\title{
The pion structure function and jet production in
} $\gamma p \rightarrow n X$

\section{Michael Klasen}

II. Institut für Theoretische Physik, Universität Hamburg, Luruper Chaussee 149, D-22761 Hamburg, Germany

\begin{abstract}
.
Despite its theoretical and practical importance, the pion structure is still badly constrained, particularly at low $x_{\pi}$ and in the sea-quark and gluon sectors. Recently ZEUS have presented data on dijet photoproduction with a leading neutron, which is dominated by slightly off-shell pion exchange and can be used to constrain the pion densities down to $x_{\pi} \simeq 0.01$. We compare a recent NLO calculation to the ZEUS data and find that the lower gluon densities of SMRS seem to be preferred by the data. Theoretical uncertainties, in particular from the pion flux, are discussed in some detail.
\end{abstract}




\section{Introduction}

As one the simplest QCD bound states and as the Goldstone boson of chiral symmetry breaking, the pion is a very intersting theoretical object: Its structure carries important implications for the QCD confinement mechanism and the realization of symmetries like isospin in nature. It is also of practical importance for the hadronic input to the photon structure at low scales. The latter is connected via Vector Meson Dominance to the $\rho$ meson structure, which is poorly known and thus often replaced by the pion structure.

Unfortunately, determinations of the pion structure have made little progress over the last decade. They are based on old Drell-Yan and prompt photon data at fixed target energies and large values of the partonic momentum fraction $x_{\pi}$. Many details are still based on pure theoretical assumptions. In order to improve the situation, it has been proposed to measure the (virtual) pion structure at low $x_{\pi}$ in deep inelastic scattering (DIS) and photoproduction with leading neutrons at HERA [1]. Since the pion is by far the lightest hadron, its exchange dominates the $p \rightarrow n$ transition and it will almost be on its mass shell, particularly at small values of the squared momentum transfer $t^{\prime}$ between the proton and the neutron.

Recently the H1 and ZEUS collaborations at HERA have installed forward proton and neutron calorimeters and presented first results on the semi-inclusive DIS structure

functions $F_{2}^{\mathrm{LP}(3)}\left(\beta, x_{p}, Q^{2}\right)$ and $F_{2}^{\mathrm{LN}(3)}\left(\beta, x_{n}, Q^{2}\right)$ [2, 3] and on dijet photoproduction [4, 5]. The latter has recently been compared to a next-to-leading order (NLO) calculation [6]. In Sec. 2 of this paper, we define the dijet photoproduction cross section, the kinematic variables, and the experimental conditions of the scattering process. In Sec. 3 we discuss the pion flux and in Sec. 1 the pion structure. The main results are contained in Sec. 5, where we compare our NLO dijet calculation with the ZEUS data. A brief summary is given in Sec. 6 .

\section{Kinematics}

The production of two jets with a leading neutron at HERA is shown schematically in Fig. 11. The corresponding cross section

$$
\frac{\mathrm{d}^{3} \sigma}{\mathrm{d} E_{T}^{2} \mathrm{~d} \eta_{1} \mathrm{~d} \eta_{2}}=\sum_{a, b} x_{a} f_{a / e}\left(x_{a}, M_{\gamma}^{2}\right) x_{b} f_{b / p}\left(x_{b}, M_{\pi}^{2}\right) \frac{\mathrm{d} \hat{\sigma}_{a b \rightarrow 12}}{\mathrm{~d} t}
$$

depends on the partonic cross section $\mathrm{d} \hat{\sigma}_{a b \rightarrow 12} / \mathrm{d} t$ and on the parton densities in the electron and proton

$$
\begin{aligned}
& f_{a / e}\left(x_{a}, M_{\gamma}^{2}\right)=\int_{x_{a}}^{1} \frac{\mathrm{d} y}{y} f_{a / \gamma}\left(x_{\gamma}, M_{\gamma}^{2}\right) f_{\gamma / e}\left(y, Q^{2}\right) \\
& f_{b / p}\left(x_{b}, M_{\pi}^{2}\right)=\int_{x_{b}}^{1} \frac{\mathrm{d}\left(1-x_{n}\right)}{1-x_{n}} f_{b / \pi}\left(x_{\pi}, M_{\pi}^{2}\right) f_{\pi / p}\left(1-x_{n}, t^{\prime}\right) .
\end{aligned}
$$

Both are convolutions of flux factors and parton densities. The photon flux $f_{\gamma / e}\left(y, Q^{2}\right)$ is given by the usual Weizsäcker-Williams approximation [7]. The ranges of the photon momentum fraction $y=(P \cdot q) /(P \cdot k) \in[0.2 ; 0.8]$ and virtuality $Q^{2}=-q^{2}<4 \mathrm{GeV}^{2}$ 


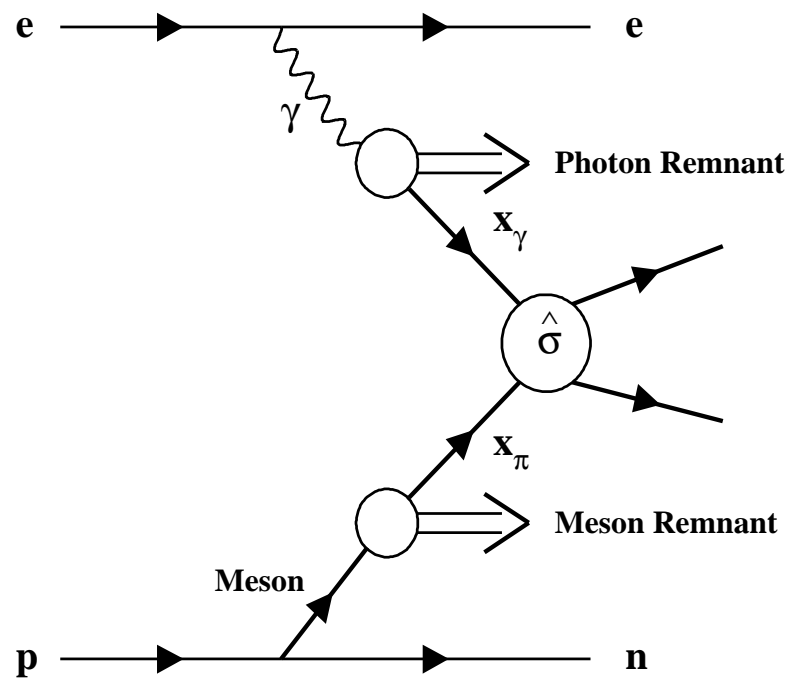

Figure 1. Schematic diagram for dijet production with a leading neutron in photoproduction at HERA.

are dictated by the ZEUS experiment. The momentum fraction of the leading neutron $x_{n}$ and the momentum transfer $t^{\prime}$ in the pion flux factor $f_{\pi / p}\left(1-x_{n}, t^{\prime}\right)$ are restricted to $x_{n}=\left(P^{\prime} \cdot k\right) /(P \cdot k)>400 \mathrm{GeV} / 820 \mathrm{GeV}$ and $t^{\prime}=f\left(p_{T}\right)=-\frac{p_{T}^{2}}{x_{n}}-\frac{\left(1-x_{n}\right)\left(m_{n}^{2}-x_{n} m_{p}^{2}\right)}{x_{n}}$ with $p_{T}<x_{n} \cdot 0.66 \mathrm{GeV}$. The hard jets are defined with the $k_{T}$ cluster algorithm and a maximal distance $R_{i j}=\sqrt{\left(\eta_{i}-\eta_{j}\right)^{2}+\left(\phi_{i}-\phi_{j}\right)^{2}}<1$ [8]. They are required to have transverse energies above $E_{T}^{\text {jet }}>6 \mathrm{GeV}$ and lie in the central rapidity range $-2<\eta^{\text {jet }}<2$.

\section{Pion Flux}

In principle, a variety of soft reactions can lead to a leading neutron final state at HERA. Four different possibilities are shown in Fig. 2: a) charged pion exchange, b) intermediate $\Delta$ production, c) Reggeon $\left(\rho, a_{2}\right)$ exchange, and d) Pomeron exchange. Since the momentum transfer is restricted to low values $t^{\prime}<0.54 \mathrm{GeV}^{2}$ in the ZEUS

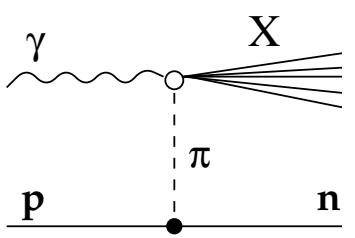

a

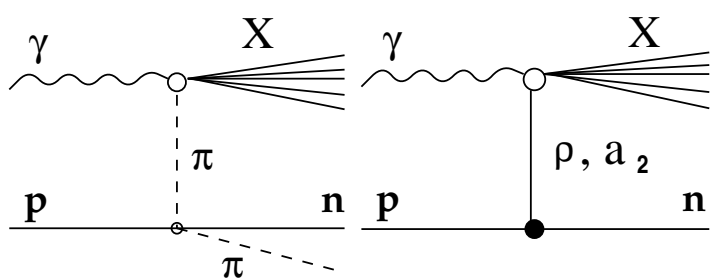

b

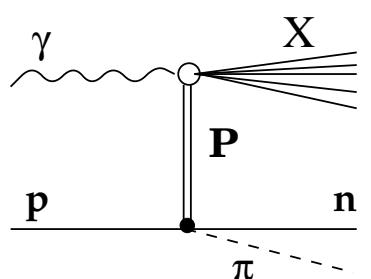

d

Figure 2. Photoproduction with a leading neutron: a) Charged pion exchange, b) intermediate $\Delta$ production, c) Reggeon exchange, and d) Pomeron exchange. 

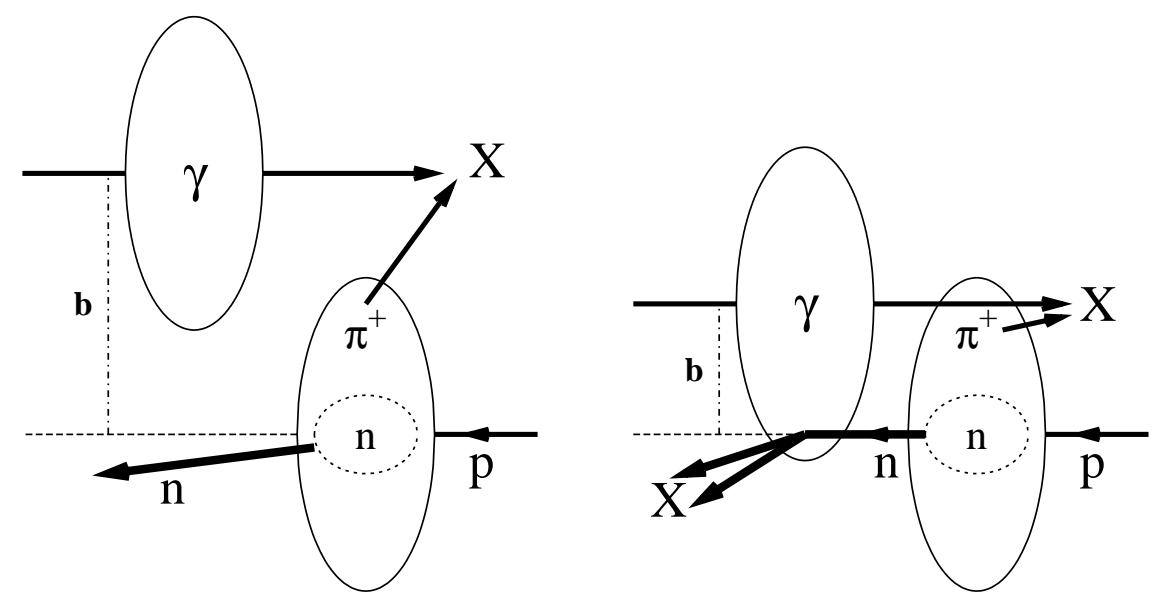

Figure 3. Photoproduction with a leading neutron in the meson cloud model at large (left) and small (right) impact parameter $b$.

experiment, the pion as the lightest particle in the $t^{\prime}$-channel dominates. Diagrams, where an additional pion is produced in the proton fragmentation region (diagrams b) and $\mathrm{d})$ ), are experimentally excluded. Reggeon exchange amounts to a small $(<10 \%)$ cross section increase.

In the meson cloud model, the photon-proton collision can be viewed as a process, where a pionic cloud is stripped off a neutron core (see Fig. 3). As the impact parameter $b$ becomes small, additional effects like photon absorption or neutron-rescattering may become important. The semi-inclusive ZEUS data show that absorption effects decrease the cross section by at most $10 \%$ in the $x_{n}$ range considered, which cancels approximately the increase of the cross section by Reggeon exchange.

The light cone representation of the proton wave function is given by

$|p\rangle=\sqrt{Z}\left[\left|p_{0}\right\rangle+\int \mathrm{d} x \mathrm{~d}^{2} \mathbf{p}_{\mathbf{T}}\left(\phi_{\pi / p}\left(x, \mathbf{p}_{\mathbf{T}}\right) \sqrt{\frac{1}{3}}\left|p, \pi^{0}\right\rangle+\phi_{\pi / p}\left(x, \mathbf{p}_{\mathbf{T}}\right) \sqrt{\frac{2}{3}}\left|n, \pi^{+}\right\rangle+\ldots\right)\right]$,

where $\left|p_{0}\right\rangle$ is the bare proton wave function, given by a three-quark state, and $\phi_{M / p}\left(x, \mathbf{p}_{\mathbf{T}}\right)$ is the probability amplitude of finding a baryon $\mathrm{B}(=\mathrm{p}, \mathrm{n})$ with longitudinal momentum fraction $x$ and transverse momentum $\mathbf{p}_{\mathbf{T}}$ and a meson $\mathrm{M}\left(=\pi^{0}, \pi^{+}\right)$in the proton. $Z$ is the wave function renormalization constant and is fixed by the normalization condition $\langle p \mid p\rangle=1$. The splitting function or flux factor takes the general form

$$
\begin{aligned}
f_{\pi / p}\left(1-x_{n}, t^{\prime}\right) & =\int \mathrm{d}^{2} \mathbf{p}_{\mathbf{T}}\left|\phi_{\pi / p}\left(x_{n}, \mathbf{p}_{\mathbf{T}}\right)\right|^{2} \delta\left(t^{\prime}-f\left(p_{T}\right)\right) \\
& =\frac{3 C_{n}}{4 \pi} \frac{g_{n \pi p}^{2}}{4 \pi} \frac{-t^{\prime}}{\left(m_{\pi}^{2}-t^{\prime}\right)^{2}}\left(1-x_{n}\right)^{1-2 \alpha_{\pi}^{\prime}\left(t^{\prime}-m_{\pi}^{2}\right)}\left[F\left(x_{n}, t^{\prime}\right)\right]^{2} .
\end{aligned}
$$

It can be calculated in covariant or time-ordered perturbation theory. $C_{n}$ is the squared Clebsch-Gordon isospin coefficient (2/3 for leading neutrons, 1/3 for leading protons), $1 /\left(m_{\pi}^{2}-t^{\prime}\right)^{2}$ is the squared pion propagator, and $\left(1-x_{n}\right)^{1-2 \alpha_{\pi}^{\prime}\left(t^{\prime}-m_{\pi}^{2}\right)}$ accounts for the 
The pion structure function and jet production in $\gamma p \rightarrow n X$

\begin{tabular}{|c|c|c|c|c|c|c|c|c|}
\hline Group & Year & Set & $\begin{array}{c}Q_{0}^{2} \\
\left(\mathrm{GeV}^{2}\right)\end{array}$ & $\begin{array}{l}\text { Fac. } \\
\text { Sch. }\end{array}$ & Model & Data & $N_{f}$ & $\begin{array}{l}\Lambda_{\overline{\mathrm{MS}}}^{N_{f}=4} \\
(\mathrm{MeV})\end{array}$ \\
\hline ABFKW & 1989 & $\mathrm{NLO}$ & 2.00 & $\overline{\overline{\mathrm{MS}}}$ & $\begin{aligned} v^{\pi} & =\gamma X, \mathrm{DY} \\
s^{\pi} & =\mathrm{DY} \\
g^{\pi} & =\gamma X, \mathrm{MSR}\end{aligned}$ & $\begin{array}{l}\text { WA70,NA24 } \\
\text { NA3 } \\
\text { WA70,NA24 }\end{array}$ & 4 & 229 \\
\hline SMRS & 1992 & $\begin{array}{l}10 \% \\
15 \% \\
20 \%\end{array}$ & 4.00 & $\overline{\mathrm{MS}}$ & $\begin{aligned} v^{\pi} & =\mathrm{DY} \\
s^{\pi} & =\mathrm{DY} \\
g^{\pi} & =\gamma X, \mathrm{MSR}\end{aligned}$ & $\begin{array}{l}\text { NA10, E615 } \\
\text { NA3 } \\
\text { WA70 }\end{array}$ & 4 & 190 \\
\hline GRV & 1992 & $\begin{array}{c}\mathrm{LO} \\
\mathrm{NLO}\end{array}$ & $\begin{array}{l}0.25 \\
0.30\end{array}$ & $\frac{\mathrm{LO}}{\mathrm{MS}}$ & $\begin{aligned} v^{\pi} & =\mathrm{ABFKW} \\
s^{\pi} & =0 \\
g^{\pi} & =\mathrm{MSR}\end{aligned}$ & WA70,NA24 & 6 & 200 \\
\hline GRSc & 1999 & $\begin{array}{c}\mathrm{LO} \\
\mathrm{NLO}\end{array}$ & $\begin{array}{l}0.26 \\
0.40\end{array}$ & $\frac{\mathrm{LO}}{\mathrm{MS}}$ & $\begin{aligned} v^{\pi} & =\mathrm{DY}, \mathrm{MSR} \\
s^{\pi} & =\left(v^{\pi} / v^{p}\right) s^{p} \\
g^{\pi} & =\left(v^{\pi} / v^{p}\right) g^{p}\end{aligned}$ & $\begin{array}{l}\text { NA10, E615 } \\
\text { H1, ZEUS } \\
\text { H1, ZEUS }\end{array}$ & 3 & $\begin{array}{l}204 \\
299\end{array}$ \\
\hline
\end{tabular}

Table 1. Parameterizations of the parton densities in the pion. They are constrained by Drell-Yan (DY) and prompt photon production $(\gamma X)$ data and by the Momentum Sum Rule (MSR).

virtuality and possible reggeization of the pion. The interaction term in the pion-nucleon Lagrangean leads to the numerator $-t^{\prime}$, and off-mass shell effects of the higher $\left|p, \pi^{0}\right\rangle$ and $\left|n, \pi^{+}\right\rangle$Fock states are modeled by a form factor

$$
F\left(x_{n}, t\right)=\left\{\begin{array}{lr}
\exp \left[b\left(t-m_{\pi}^{2}\right)\right] & \text { [Exponential] } \\
\exp \left[R^{2}\left(t-m_{\pi}^{2}\right) /\left(1-x_{n}\right)\right] & \text { [LightCone] }
\end{array}\right.
$$

The flux factor is constrained by a symmetry relation $\left(f_{\pi / p}\left(1-x_{n}, t^{\prime}\right)=f_{n / p}\left(x_{n}, t^{\prime}\right)\right)$ and charge and momentum sum rules. Since the momentum transfer $t^{\prime}$ is small, we neglect reggeization $\left(\alpha^{\prime}=0\right)$ and choose a light cone form factor with $R=0.5 \mathrm{GeV}^{-1}$ in good agreement with recent determinations [9, 10]. The pion-nucleon coupling constant $g^{2} /(4 \pi)=14.17$ is taken from a recent extraction from the Goldberger-Miyazawa-Oehme sum rule [1].

\section{Pion Structure}

Traditionally, the valence quark densities in the pion have been determined in the DrellYan process, while the gluon density has been constrained in prompt photon production (see Tab. 1). In both cases the relevant data sets were taken in fixed target collisions in the 1980s and are restricted to large $x_{\pi} \geq 0.2$. Furthermore, they suffer from nuclear effects and fragmentation contributions, respectively, and also from scale uncertainties. The situation is worst for the sea quarks where theoretical assumptions vary most 


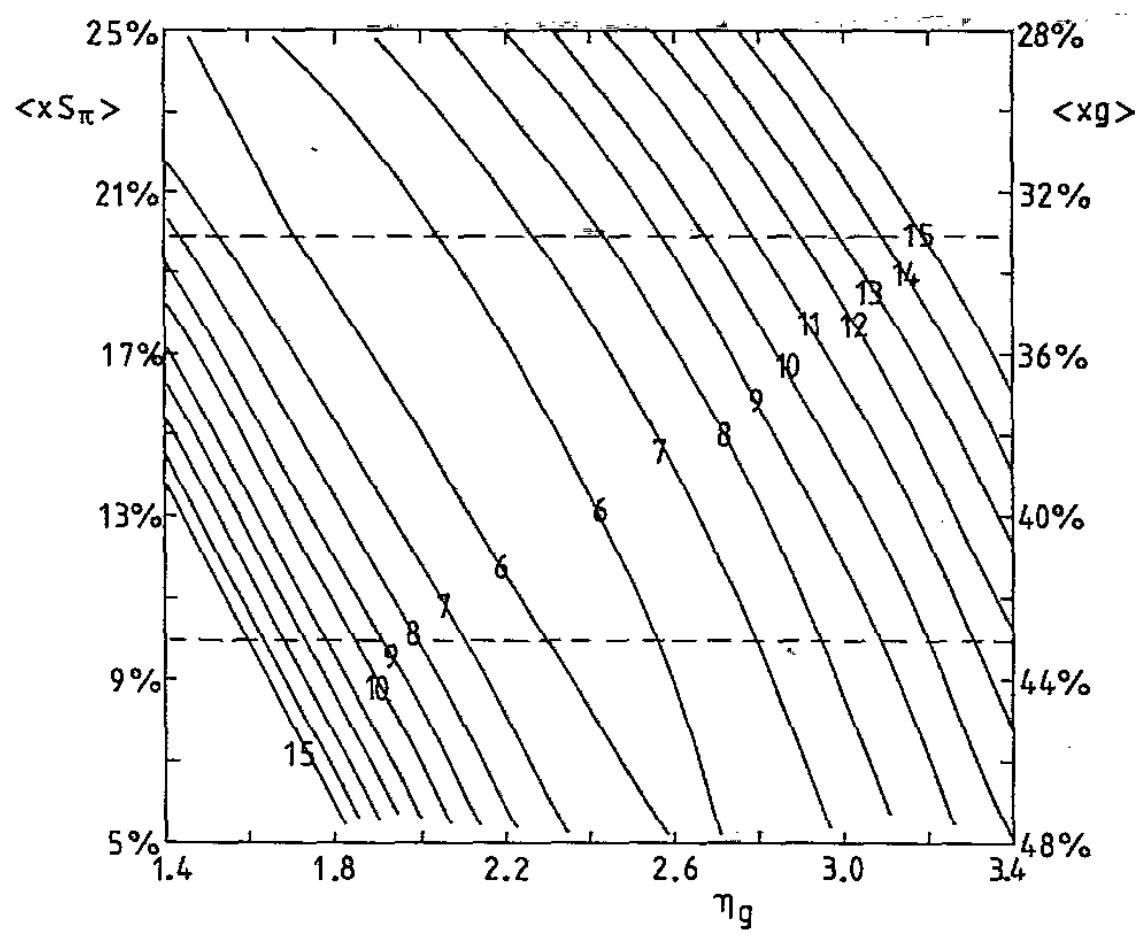

Figure 4. Momentum fractions carried by sea-quarks and gluons in the pion as determined in the SMRS fit. The lowest $\chi^{2}$ values (6-15) are obtained with sea quark momentum fractions between 10 and $20 \%$. 14.

widely. It is known that at $Q^{2}=4 \mathrm{GeV}^{2}$ valence quarks carry about half of the pion's momentum. At the same scale gluons carry about half of a proton's momentum. If pions and protons had a similar structure, as assumed by GRSc [12, no room would be left for the sea quarks. Indeed GRV assumed a zero-input density for sea quarks, however at a very low scale [13]. SMRS determined that sea quarks should however carry between 10 and $20 \%$ of the pion's momentum (see Fig. (1) 14. In Fig. 5 we compare the available parton densities in the pion at a scale $Q^{2}=E_{T, \text { min }}^{2}=36 \mathrm{GeV}^{2}$ relevant for dijet photoproduction on a slightly virtual pion. As one can see, the SMRS sea quarks and gluons turn out lower than the very similar ABFKW, GRV, and GRSc distributions [15].

\section{Dijet Production}

The ZEUS collaboration have compared their dijet results with a leading neutron with LO Monte Carlo predictions and found reasonable agreement. It is, however, wellknown that pure LO results are strongly scale dependent, that they can be modified by potentially large NLO corrections, and that they do not allow for the implementation of a jet algorithm. For inclusive dijet photoproduction, four different NLO calculations have been performed using different phase space slicing methods and the subtraction method. They all agree well within numerical errors [16]. We have implemented in our 


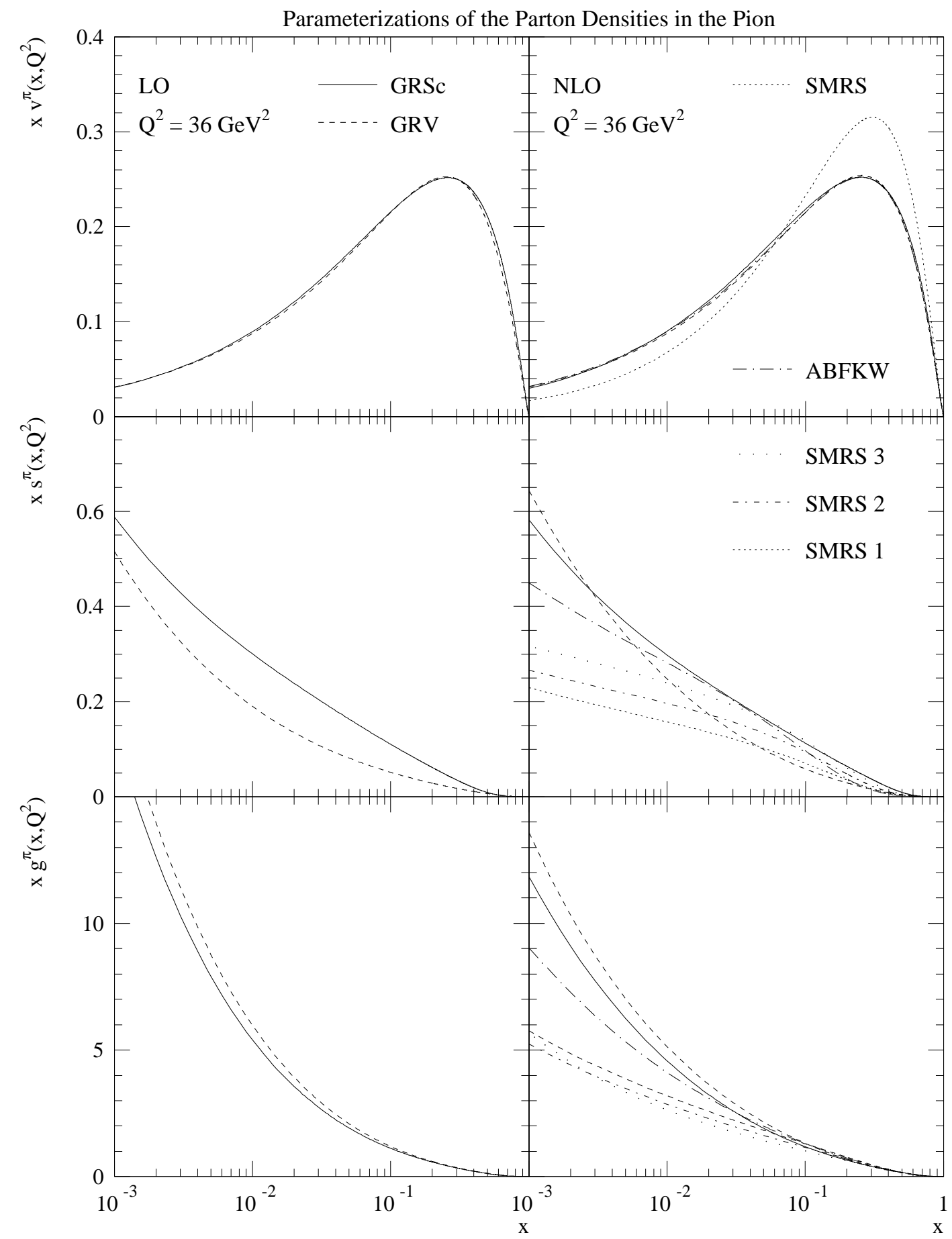

Figure 5. Parameterizations of the valence-quark (top), sea-quark (center), and gluon (bottom) densities in the pion in LO (left) and NLO (right) at $Q^{2}=36 \mathrm{GeV}^{2}$. 


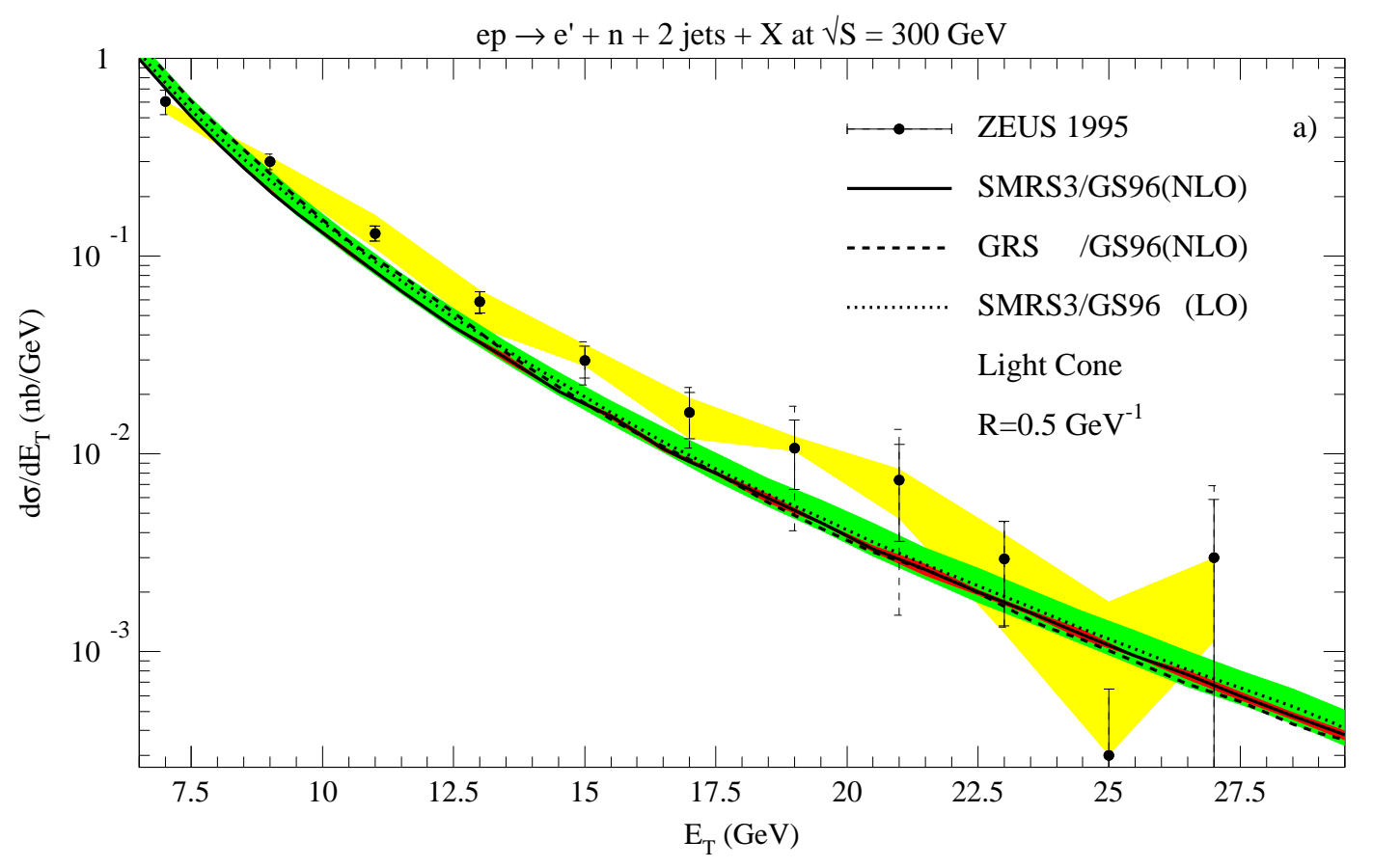

Figure 6. Dependence of the dijet photoproduction cross section with a leading neutron on the transverse energy of one of the two jets. Two NLO predictions with different pion structure functions and one LO prediction are shown together with experimental data from ZEUS with statistical (inner) and systematic (outer) experimental error bars. In addition, error bands from the theoretical scale uncertainty (medium: LO, dark: NLO) and the experimental energy scale uncertainty (light) are shown. The NLO error band coincides with the thickness of the full line.

calculation [17] two additional integrations, i.e. over $x_{n}$ and $t^{\prime}$, and the pion flux factor. The proton parton densities have been replaced with those in the pion.

Selecting the two jets with highest $E_{T}$ and integrating over the $\eta \in[-2 ; 2]$, we compare in Fig. 6 the LO and NLO $E_{T}$ distribution with GS96 photon densities [18 and two different pion densities to the ZEUS data. Almost identical results are obtained with GRV photon densities. [19] The $K$-factor turns out to be one, but the scale dependence is reduced considerably from LO to NLO. The normalization is sensitive to the chosen pion flux factor: If one includes the Regge trajectory $\left(\alpha^{\prime}=1 \mathrm{GeV}^{-2}\right)$ and omits the exponential, the cross section is reduced by $15 \%$. The same reduction is obtained with a light cone form factor and $R=0.6 \mathrm{GeV}^{-1}$. Resolved photons and gluons in the pion are important at low $E_{T}<9 \mathrm{GeV}$ and $E_{T}<22 \mathrm{GeV}$, respectively. In the ratio of leading neutron and inclusive dijet cross sections in Fig. 7, many uncertainties from the scale choice, photon densities, and hadronic energy scale cancel. The $E_{T}$ shape is then sensitive to the pion densities and shows a preference for SMRS3 over GRSc.

If the leading neutron cross section is integrated over the two highest jet $E_{T}>$ 


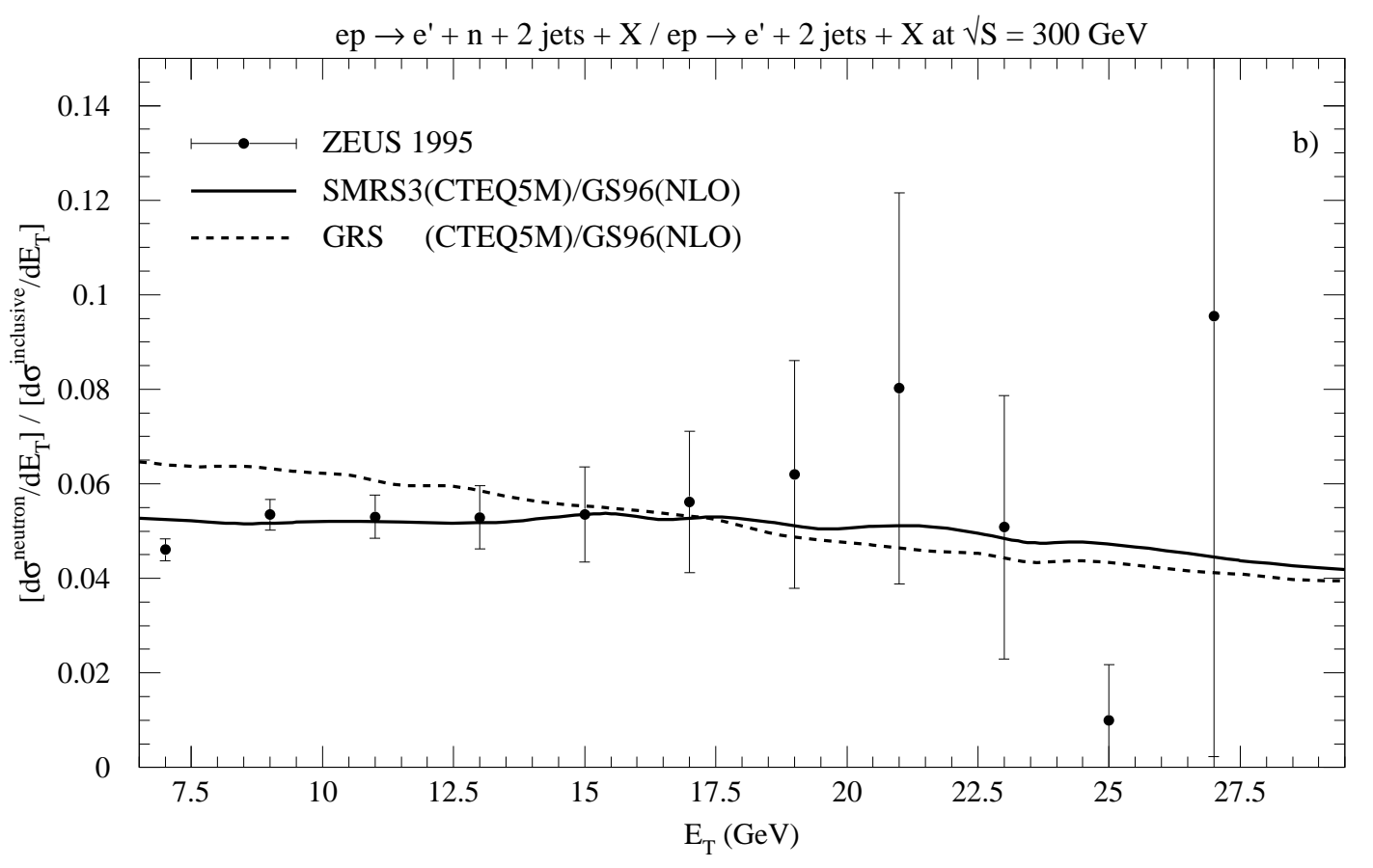

Figure 7. Ratio of the leading neutron over the inclusive cross section versus the transverse energy. NLO calculations with two different pion structure functions are shown together with the experimental ZEUS data. Only statistical error bars are given.

$6 \mathrm{GeV}$, but not over $\eta$, one obtains the rapidity distribution shown in Fig. 8. Direct photons and quarks in the photon are important at $\eta<0$. In this region of jet photoproduction, the low quarks in the NLO GS96 parameterization usually give the best fit, despite the fact that they do not reproduce the photon structure function. [20] Indeed GS96 also gives the best fit for dijets with a leading neutron. At $\eta<0$ hadronization corrections become also important and can amount to a factor of two. The data for the ratio of leading neutron over inclusive rapidity distributions in Fig. 9 have still rather large statistical error bars and do not distinguish between different pion parameterizations.

The distribution in the observed momentum fraction of the partons in the pion

$$
x_{\pi}^{\mathrm{OBS}}=\frac{E_{T_{1}} e^{\eta_{1}}+E_{T_{2}} e^{\eta_{2}}}{2\left(1-x_{n}\right) E_{p}}
$$

is shown in Fig. 10. It suffers from the fact that ZEUS have applied equal $E_{T}$ cuts on both hard jets, which is known to be infrared sensitive [21]. Therefore the cut on the second jet in the NLO calculation has to be relaxed to $E_{T, 2}>5$ or $5.5 \mathrm{GeV}$. The best fit is again obtained with GS96 photon densities and SMRS3 pion densities, which have the lowest quark and gluon distributions, respectively. It should be kept in mind that the quark densities in the photon are correlated with the gluon density in the pion. In 


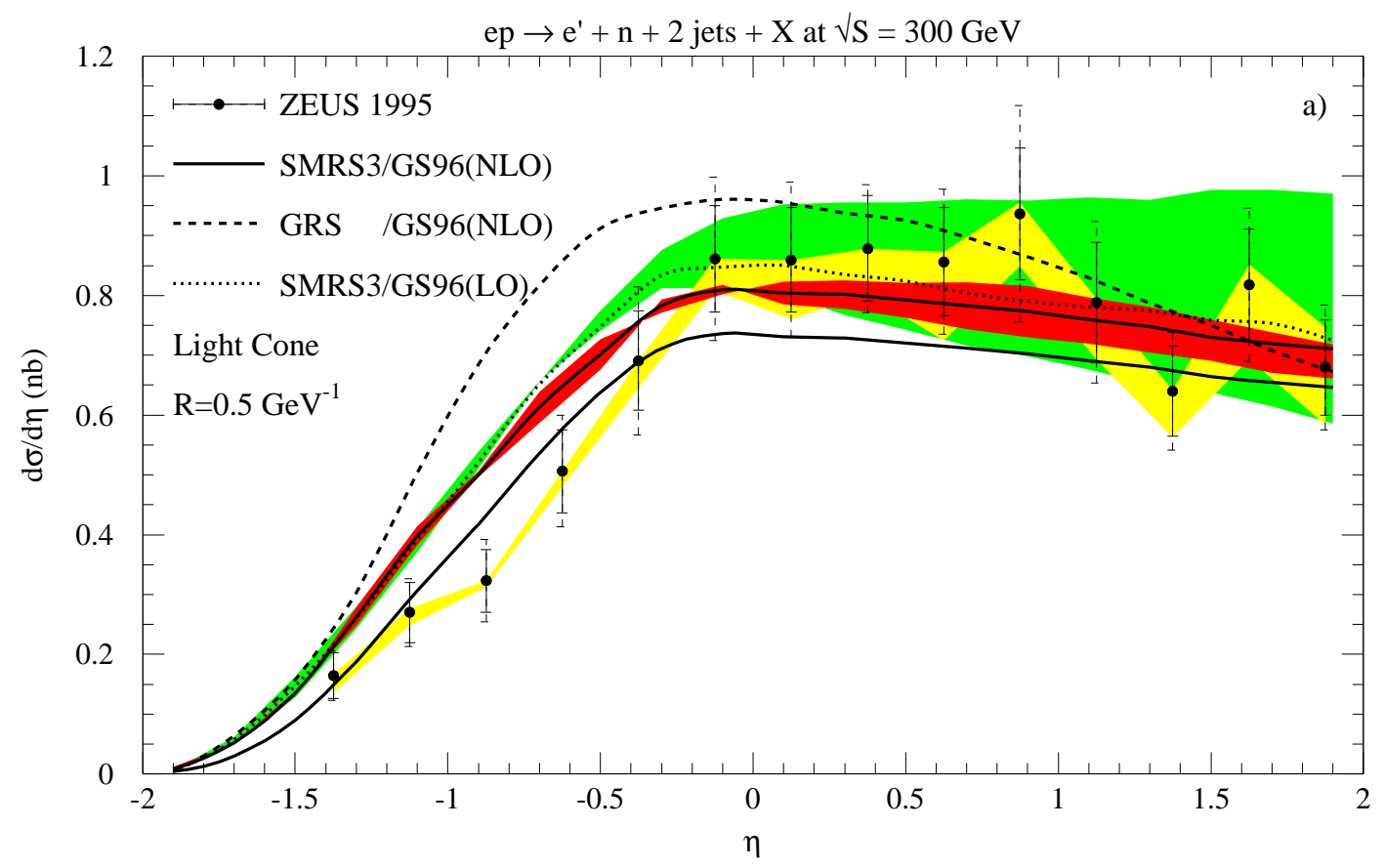

Figure 8. Dependence of the dijet photoproduction cross section with a leading neutron on the rapidity of one of the two jets. Details are as in Fig. 6. The lower full curve demonstrates the influence of hadronization corrections.

the $x_{\pi}$-distribution, hadronization corrections may play an important role at small $x_{\pi}$, which is related to negative rapidities.

\section{Summary}

Despite their theoretical and practical importance, the parton densities in the pion are still poorly constrained, particularly below $x_{\pi}<0.2$. An intriguing possibility for improvement consists in dijet photoproduction with a leading neutron at HERA, which is dominated by slightly off-shell pion exchange. Here the pion can be probed down to $x_{\pi} \simeq 0.01$. However, theoretical uncertainties from Reggeon exchange and photon absorption, modeled by a pion flux factor, have to be well under control.

Data on dijet photoproduction with a leading neutron have been obtained by the ZEUS collaboration. Unfortunately, ZEUS have applied theoretically unsafe $E_{T}$ cuts. Furthermore, the data suffer from large hadronization corrections in the backward region. On the other hand, the hard scattering cross section has been calculated in NLO and is theoretically stable. A comparison of the NLO calculation with data indicates that lower gluon densities in the pion, as predicted by the SMRS3 parameterization, are preferred over steeper gluons as predicted by ABFKW, GRV, or GRSc. 


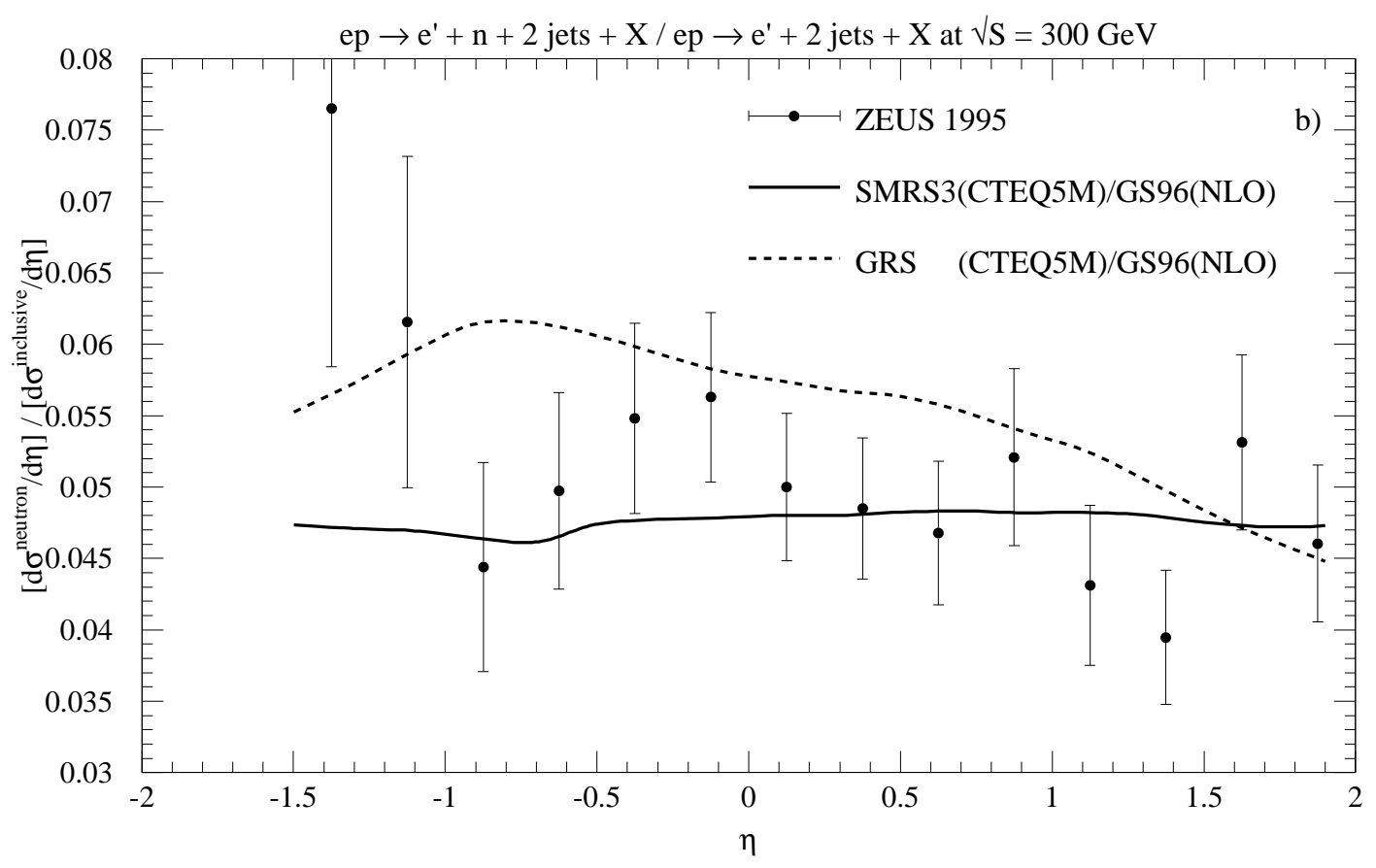

Figure 9. Ratio of the leading neutron over the inclusive cross section versus the rapidity. Details are as in Fig. 7 .

\section{Acknowledgments}

I wish to thank the organizers of the Ringberg Workshop for the kind invitation, A. Vogt for useful discussions, and G. Kramer for his collaboration. Financial support by the Deutsche Forschungsgemeinschaft through Grant No. KL 1266/1-1, by the Bundesministerium für Bildung und Forschung through Grant No. 05 HT9GUA 3, and by the European Commission through the Research Training Network Quantum Chromodynamics and the Deep Structure of Elementary Particles under Contract No. ERBFMRX-CT98-0194 is gratefully acknowledged.

\section{References}

[1] H. Holtmann, G. Levman, N. N. Nikolaev, A. Szczurek and J. Speth, Phys. Lett. B 338 (1994) 363.

[2] C. Adloff et al. [H1 Collaboration], Eur. Phys. J. C 6 (1999) 587.

[3] J. Breitweg et al. [ZEUS Collaboration], Abs. 883, contributed to ICHEP 2000, Osaka, Japan.

[4] C. Adloff et al. [H1 Collaboration], Abs. 959, contributed to ICHEP 2000, Osaka, Japan.

[5] J. Breitweg et al. [ZEUS Collaboration], hep-ex/0010019.

[6] M. Klasen and G. Kramer, hep-ph/0103056.

[7] C. F. von Weizsäcker, Z. Phys. 88 (1934) 612; E. J. Williams, Phys. Rev. 45 (1934) 729.

[8] S. D. Ellis and D. E. Soper, Phys. Rev. D 48 (1993) 3160. 


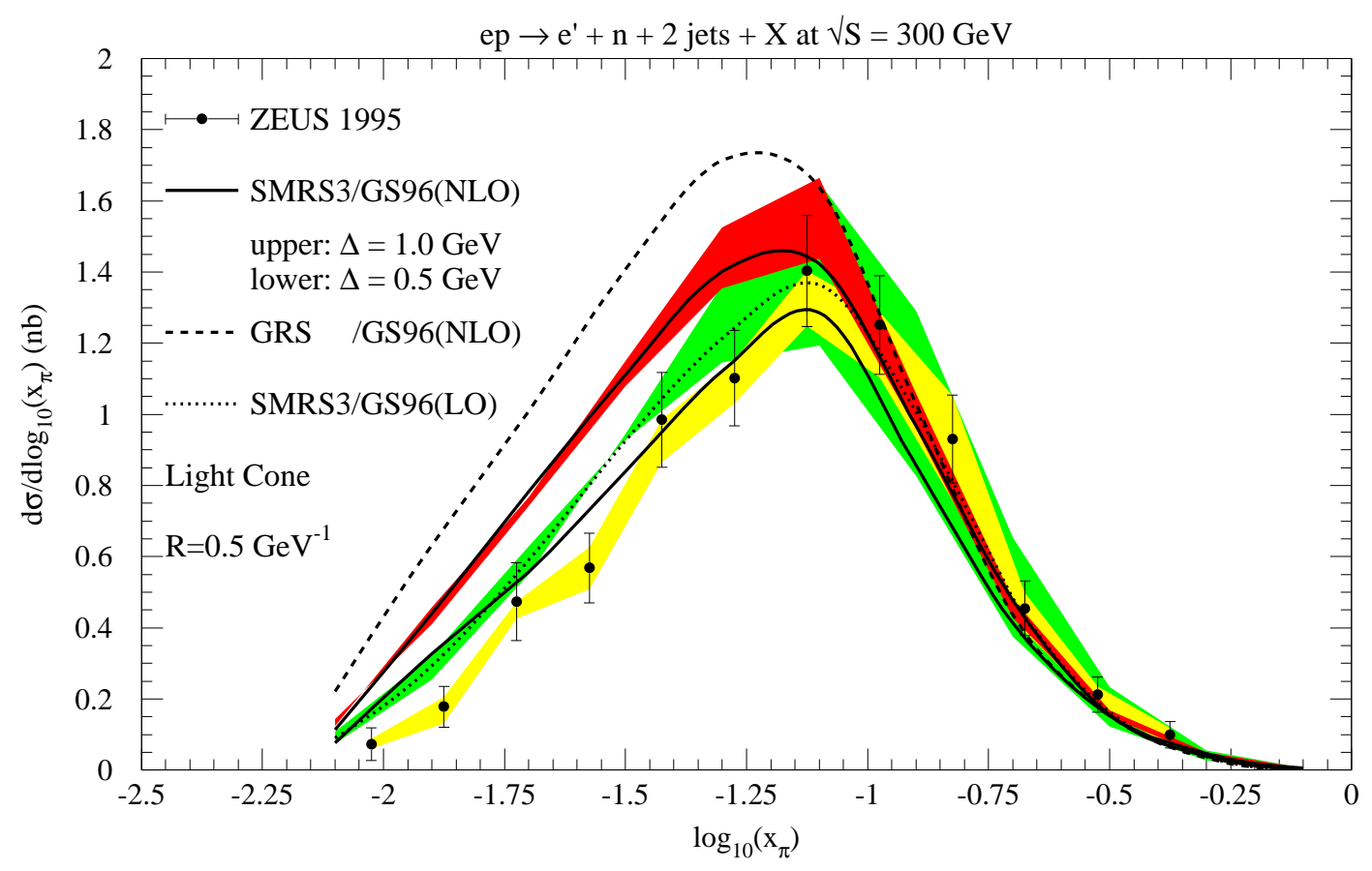

Figure 10. Dependence of the dijet photoproduction cross section with a leading neutron on the logarithm of the observed momentum fraction of the partons in the pion $\log _{10}\left(x_{\pi}\right)$. Details are as in Figs. 6 and 8 . In addition, we show the effect of lowering the transverse energy difference $\Delta$ from $1 \mathrm{GeV}$ to $0.5 \mathrm{GeV}$.

[9] H. Holtmann, A. Szczurek and J. Speth, Nucl. Phys. A 569 (1996) 631.

[10] U. D'Alesio and H. J. Pirner, Eur. Phys. J. A 7 (2000) 109.

[11] T. E. Ericson, B. Loiseau and A. W. Thomas, hep-ph/0009312.

[12] M. Glück, E. Reya and I. Schienbein, Eur. Phys. J. C 10 (1999) 313.

[13] M. Glück, E. Reya and A. Vogt, Z. Phys. C 53 (1992) 651.

[14] P. J. Sutton, A. D. Martin, R. G. Roberts and W. J. Stirling, Phys. Rev. D 45 (1992) 2349.

[15] P. Aurenche, R. Baier, M. Fontannaz, M. N. Kienzle-Focacci and M. Werlen, Phys. Lett. B 233 (1989) 517.

[16] B. W. Harris, M. Klasen and J. Vossebeld, hep-ph/9905348.

[17] M. Klasen and G. Kramer, Z. Phys. C 76 (1997) 67; M. Klasen, T. Kleinwort and G. Kramer, Eur. Phys. J. directC 1 (1998) 1.

[18] L. E. Gordon and J. K. Storrow, Nucl. Phys. B 489 (1997) 405.

[19] M. Glück, E. Reya and A. Vogt, Phys. Rev. D 46 (1992) 1973.

[20] A. Vogt, hep-ph/9709345.

[21] M. Klasen and G. Kramer, Phys. Lett. B 366 (1996) 385. 